\title{
Rupture of a Posterior Fossa Dermoid Cyst Overlying the Torcular with Extracranial Extension: Technical Note
}

\author{
0. Coulibaly1,2, E. Komi'1, L. Rifi'1, Y. Sogoba1, M. Dama², 0. Diallo², A. Melhaoui', \\ M. Boutarbouch'1, Y. Arkha' ${ }^{1}$, S. Derraz ${ }^{1}$, A. El Ouahabi' ${ }^{1}$ A. El Khamlichi1 \\ ${ }^{1}$ Hopital des Spécialités, Rabat, Morocco \\ ${ }^{2}$ Hopital du Mali, Bamako, Mali \\ Email: coulibalynch1@gmail.com
}

Received 2 January 2015; accepted 28 March 2015; published 31 March 2015

Copyright (C) 2015 by authors and Scientific Research Publishing Inc.

This work is licensed under the Creative Commons Attribution International License (CC BY). http://creativecommons.org/licenses/by/4.0/

c) (i) Open Access

\begin{abstract}
Intracranial dermoid cysts are congenital benign neoplasms mostly diagnosed in the pediatric hood and usually involve the midline structures. They count approximately less than $1 \%$ of all intracranial neoplasms and are believed to arise from ectopic cell rests incorporated in the closing neural tube. These dermoid cysts, especially those involving the posterior fossa and overlying the torcular, are uncommon. We report perhaps the first case of this entity in a third-year-old boy and discuss the physiopathogenesis, the imaging features and the best technical note to manage this cyst in this location.
\end{abstract}

\section{Keywords}

Dermoid Cyst, Torcular, Angio Mri, Technical Note

\section{Introduction}

Cranial dermoid cysts are congenital neoplasms mostly diagnosed in the pediatric hood and usually involve the midline structures, especially in the supra- and parasellar region and the posterior fossa [1]. They are usually excised by pediatric surgeons because they become enlarged, infected, discharged fluid or for cosmetic reasons, or to allay parental anxiety [2]. Because of their association with an intracranial extension, it has been advocated that all cranial dermoid cysts should be excised by neurosurgeons and that all children with midline lesions should undergo computed tomography (CT) scanning to exclude an intracranial extension [3]. Intracranial dermoid cysts count approximately less than $1 \%$ of all intracranial neoplasms and are believed to arise from ectopic 
cell rests incorporated in the closing neural tube in the third to fifth week of embryonic development [4]. These intracranial dermoid cysts, especially those involving the posterior fossa and overlying the torcular, are uncommon. We report perhaps the first case of this entity in a third old boy and discuss the pathogenesis, the imaging and the best therapeutic management in this location.

\section{Case Report}

A 3-year-old boy, with no past medical history, had been admitted within our department for a 5-month history of progressive occipital tumefaction centred on the external occipital protuberance. In interrogatory, this tumefaction was preceded of a discharged clear fluid few days before with low-grade fever and treated like meningitis without results by several general praticians. For cosmetic reason and to allay parental anxiety, this boy was referred to our department formore investigations. On admission, physical examination found an healthy boy consciousness, afebrile, with no deficit and presented a $5 \mathrm{~cm} \times 4 \mathrm{~cm}$ middle occipital scalp-swelling renitent and painless lesion with a very small bony defect on palpation located just below the external occipital protuberantia without discharged fluid or pus (Figure 2(a)).

Angio-MRI of the head showed a $3-4 \mathrm{~cm}$ intracranial retrovermian heterogeneous lesion, extra axial and majority hypo intenseon T1W1 images and hyper intense on T2W2 images, separated by a thin zone of bone and connected with a dermal sinus to another extracranial lesion overlying the torcular. Post contrast images showedstrong rim enhancement of the masses and the sinus tract (Figure 1).

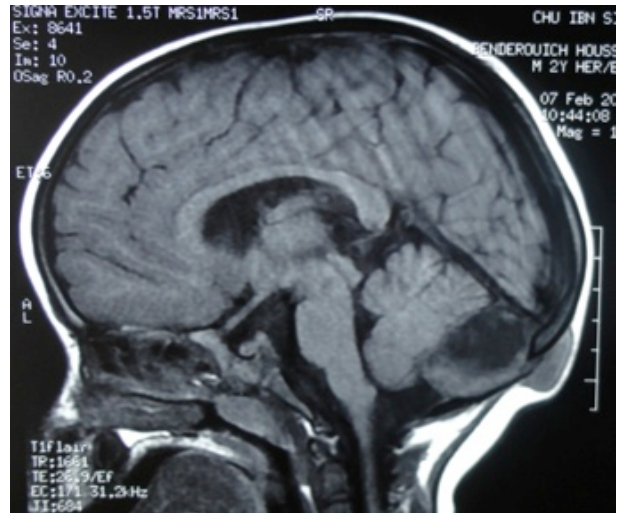

(a)

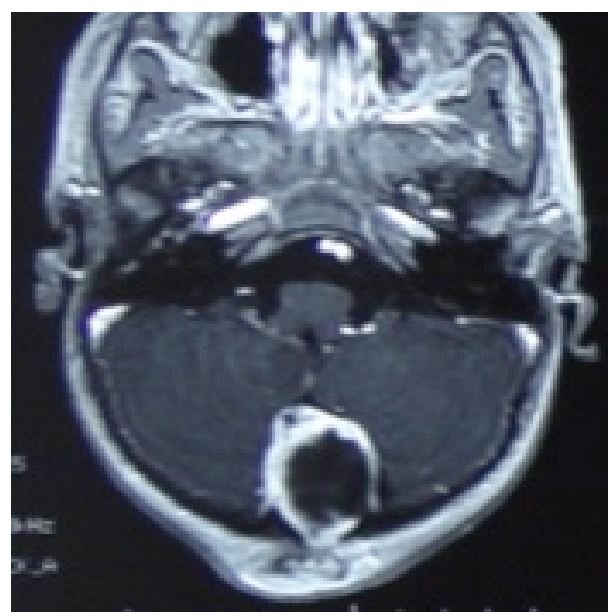

(c)

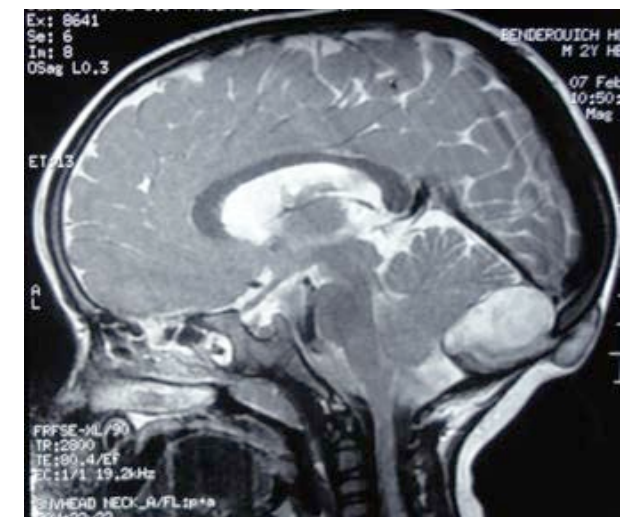

(b)

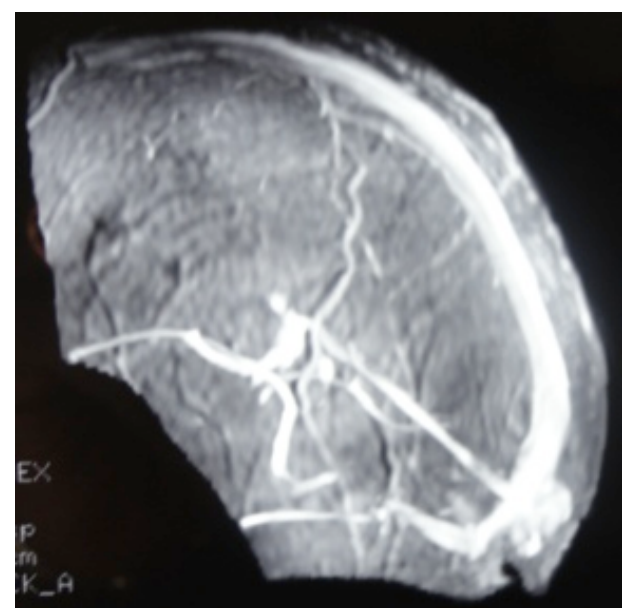

(d)

Figure 1. (a) Sagittal T1W1 MR imaging showing a hypointense PF lesion overlying the torcular with the compression of the vermis and the fourth ventricle and connecting to an extracranial component by a sinus tract. (b) T2 MRI showing the cyst a hyperintense lesion. (c) Contrast MRI showing the margin enhancement of the cyst and the sinus tract. (d) Angio view showing the mass overlying the torcular. 
Our neuroradiological team thought firstly to an infected meningocele. The result of routine blood examination was normal. Regarding the proximity of these masses with the torcular with possible adhesionsto these venial structures, and the possibility to have some vessels damages during intervention, we performeda posterior fossa craniectomy centred on the torcular that permitted to have the gross total removal of this lesion without vascular damage.We think, this technique is safe, reproducible and allows direct view and access to all the structures in this area. Peroperatively, it was seen a simple extra cranial cyst containing a clear fluid mimicking meningocele communicating to a huge intradural mixed cyst by a complete dermal sinus. This encapsulated cyst contained hairs and gelatinous lipidic formations (sebum) suggesting the diagnosis of complete intradural dermoid cyst (Figure 2). These data were confirmed by pathological investigations (Figure 3). Post operatively, an occipital meningocelae occurred and was managed by repeated lumbar punctions. He was discharged from hospital ten days after hospitalization and was free of symptoms since five years.

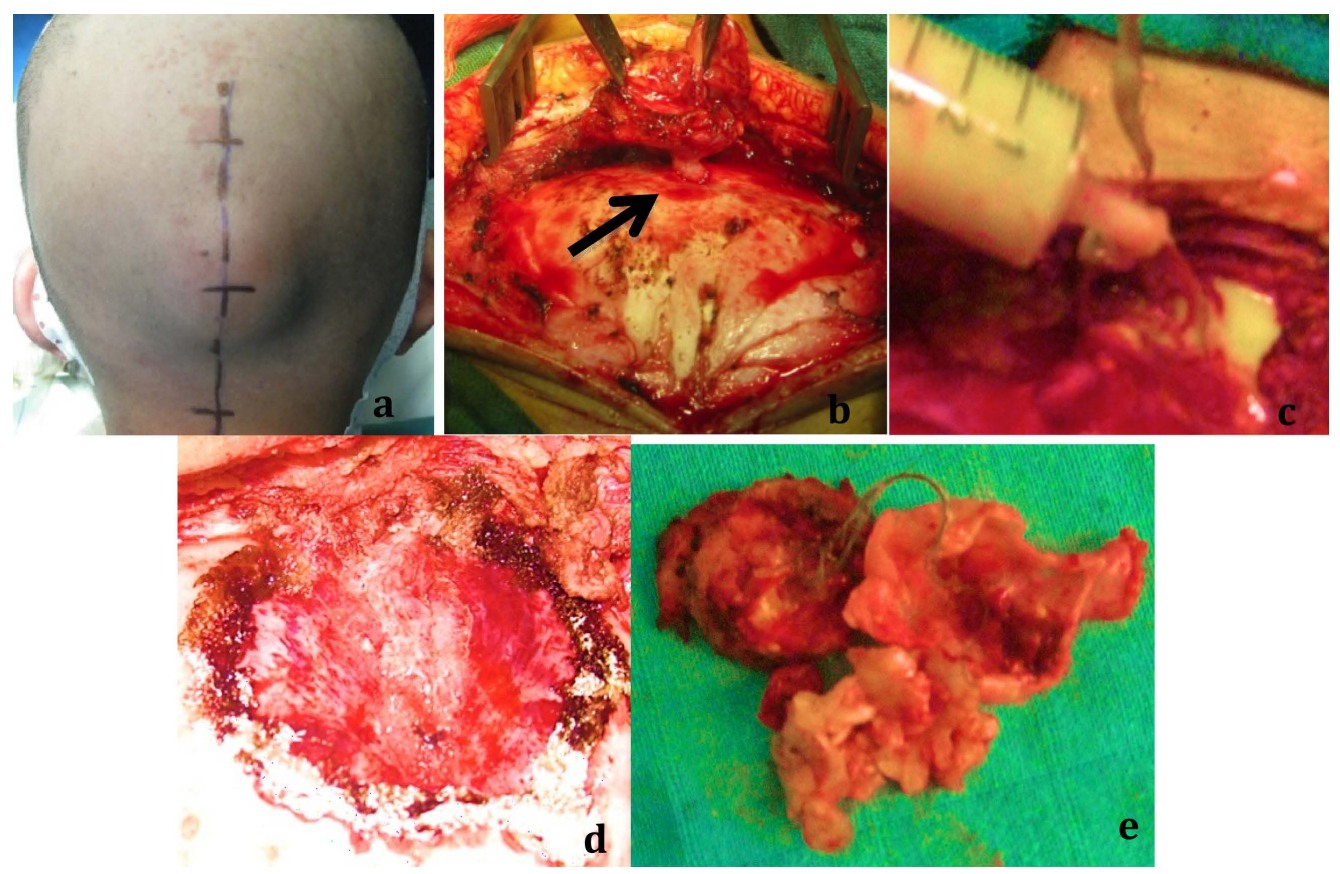

Figure 2. (a) Skin mark. (b) Dissection of the extracranial component and magnification of the sinus tract $(\boldsymbol{\lambda})$. (c) Operative view showing hair and sebum. (d) Reparation of the dura after gross total removal of this mass. (e) Encapsulated cyst with hairs and gelatinous lipidic formations (sebum).

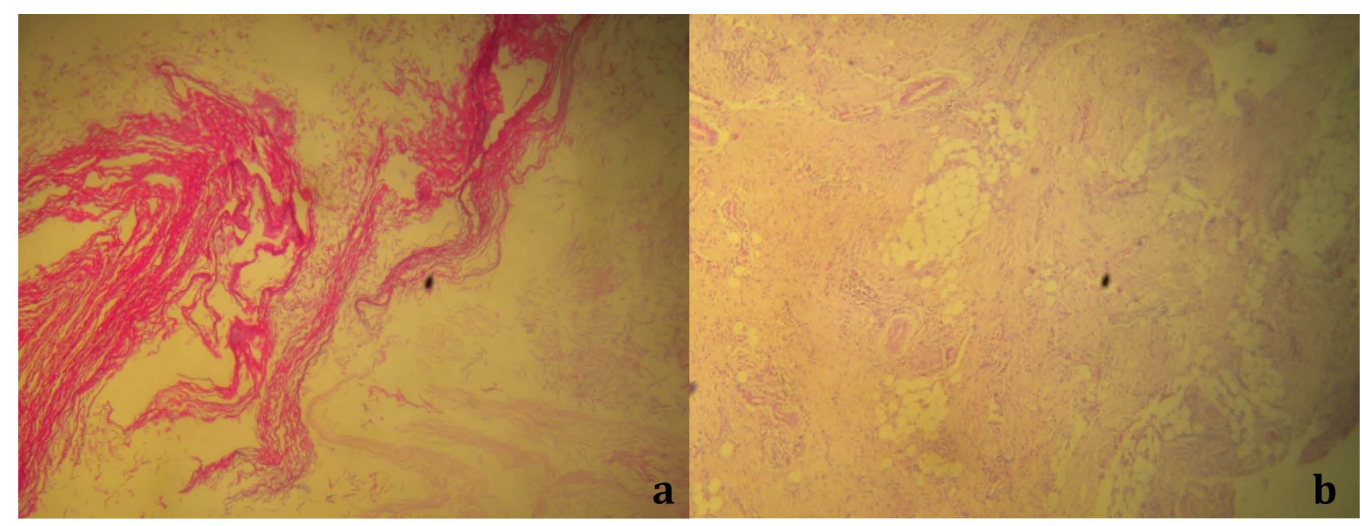

Figure 3. (a) Photomicrograph of the specimen shows a cyst wall containing stratified squamous epithelium and lamellar keratinous structure consistent with a dermoid cyst (H \& E, 200). (b) Photomicrograph of the specimen shows chronic inflammatory changes including multinucleated foreign-body giant cells (H \& E, 200). 


\section{Discussion}

Intracranial dermoid cysts account less than for $1 \%$ of intracranial tumors [2]. They result from defective closure of the neural tube during gestational weeks 3 - 5 [4]. They are benign tumors with thick capsules that are lined by squamous epithelium, which contains skin appendages (e.g., hair, sebaceous and sweat glands). The most common locations for dermoid cysts are the posterior fossa and frontal glabellar region. Posterior fossa dermoid cysts are typically midline within the vermis, but can alsooccur in the fourth ventricle. They can develop separately or associated with a dermal sinus that can be discovered at/or shortly after birth as a small cutaneous pit, or present as a subcutaneous mass.

Logue and Till [5] classified posterior fossa dermoid cysts into four groups depending on whether they were extradural or intradural, and on the degree of development of the dermal sinus, whether absent, partial, or complete: 1) extradural dermoid cyst with a complete sinus; 2) intradural dermoid cyst without a dermal sinus; 3 ) an intradural dermoid cyst with an incomplete dermal sinus; 4) intradural dermoid cyst with a complete dermal sinus. Our case was an intradural dermoid cyst with a complete dermal sinus (Figure 2).

However, the exact pathophysiological mechanism of dermoid cyst rupture is unknown [6]. Dermoid cystic tumor rupture usually occurs spontaneously or secondary to closed head trauma or iatrogenic surgical complications [7] [8]. Stendel and associates hypothesized that glandular secretions, possibly increased byage-dependent hormonal changes, may lead to rapid enlargement and rupture of these cysts [1]. Such rupture of this cyst leads to spillage of contents that may create an inflammatory response, chemical meningitis, recurrent meningitis, abscess formation, extradural empyema, increased intracranial pressure and seizure [6] [9] [10].

The typical neuroimaging signs of DCs are hypodensity on CT scan, high signal intensity on MR T1-weighted sequences (presence of fat inclusion), and variable signal intensity on T2-weightedsequences that ranges from hypointense to inhomogeneously hyperintense ( due for the presence of fair and sebaceous contents). Enhancement after contrast administration isuncommon, but atypical neuroimaging displays of DCs arenot infrequent. Fluid-attenuated inversion recovery images and, rarely, magnetic resonance spectroscopy may be necessary to diagnose a dermoid cyst [11]. The main differential diagnosis of cranial dermoids, particularly in midline lesions, is a small cranialmeningocele [2]. This diagnosis was advocated in our case too.

Microsurgical resection of the tumor if possible, is the treatment of choice in symptomatic dermoid cysts. Total resection should be the main goal but may not be achieved in all cases. The dermoid tumors particularly display dense reactive involvement of the arachnoid and occasionally of the pia mater. Rarely, the infiltration is too extensive and involves vital structures, which cannot be sacrificed [12]. Liu et al. suggested that residual cyst capsules adherent to neurovascular structures must be left behind to minimize the risk of complications, but they achieved gross total resection in mostcases [13]. So, during surgery, vigorous effort to remove the capsule of a dermoid cyst overlying the torcular herophili may result in rapid and fatal exsanguination as a consequence of unanticipated connection between the dermal sinus and the torcular herophili [6] [14]. In our mind and according to Wallace et al., it is not necessary to attempt complete removal of the cyst wall if it is firmly adherent to the brain structures [15]. So, an exciting and large view (craniectomy centred on the torcular) is the main technical note that permitted to do the total resection in our case.

\section{Conclusion}

A dermoid cyst underlying the torcular might be explored by the angio-MRI. A large craniectomy centred on the torcular with a careful dissection on bipolar coagulation might be a safely technical note to excise the lesion without vessel damage.

\section{Acknowledgements}

We would like to thank Mrs. Barry Najah for her supports for this manuscript.

\section{References}

[1] Stendel, R., Pietila, T.A., Lehmann, K., Kurth, R., Suess, O. and Brock, M. (2002) Ruptured Intracranial Dermoid Cysts. Surgical Neurology, 57, 391-398. http://dx.doi.org/10.1016/S0090-3019(02)00723-1

[2] Crawford, R. (1990) Dermoid Cyst of the Scalp: Intracranial Extension. Journal of Pediatric Surgery, 25, $294-295$. http://dx.doi.org/10.1016/0022-3468(90)90068-K 
[3] Lye, R.H. and Pickard, J.D. (1980) Occipital "Sebaceous Cysts"-A Trap for the Unwary. British Journal of Surgery, 67, 333-334. http://dx.doi.org/10.1002/bjs.1800670510

[4] Douvoyiannis, M., Goldman, D.L., Abbott III, I.R. and Litman, N. (2008) Posterior Fossa Dermoid Cyst with Sinus Tract and Meningitis in a Toddler. Pediatric Neurology, 39, 63-66. http://dx.doi.org/10.1016/i.pediatrneurol.2008.03.019

[5] Logue, V. and Till, K. (1952) Posterior Fossa Dermoid Cysts with Special Reference to Intracranial Infection. Journal of Neurology, Neurosurgery, and Psychiatry, 15, 1-12.

[6] Gulsen, S., Yilmaz, C., Serhat, C. and Altinors, N. (2010) Ruptured Intradiploic Dermoid Cyst Overlying the Torcular Herophili. Neurologia i Neurochirurgia Polska, 44, 308-313.

[7] Das, C.J., Tahir, M., Debnath, J. and Pangtey, G.S. (2007) Neurological Picture. Ruptured Intracranial Dermoid. Journal of Neurology, Neurosurgery, and Psychiatry, 78, 624-625. http://dx.doi.org/10.1136/jnnp.2006.109835

[8] Kim, I.Y., Jung, S., Jung, T.Y., et al. (2008) Traumatic Rupture of an Intracranial Dermoid Cyst. Journal of Clinical Neuroscience, 15, 469-471. http://dx.doi.org/10.1016/j.jocn.2006.10.028

[9] Martinez-Lage, J.F., Ramos, J., Puche, A., et al. (1997) Extradural Dermoid Tumours of the Posterior Fossa. Archives of Disease in Childhood, 77, 427-430. http://dx.doi.org/10.1136/adc.77.5.427

[10] Oursin, C., Wetzel, S.G., Lyrer, P., et al. (1999) Ruptured Intracranial Dermoid Cyst. Journal of Neurosurgical Sciences, 43, 217-220.

[11] Jordan, M.R., David, W.B., George, J.S., Kennith, F.L. and Michael, J.O. (2012) Ruptured Intracranial Dermoid Cyst. Proceedings (Baylor University. Medical Center), 25, 23-25.

[12] Çırak, B., Kıymaz, N. and Kerman, M. (2004) Cerebellar Dermoid Cysts with Hydrocephalus. Journal of Pediatric Neurology, 2, 161-164.

[13] Liu, J.K., Gottfried, O.N., Salzman, K.L., et al. (2008) Ruptured Intracranial Dermoid Cysts: Clinical, Radiographic, and Surgical Features. Neurosurgery, 62, 377-384. http://dx.doi.org/10.1227/01.neu.0000316004.88517.29

[14] Wiemer, D.R. (1988) An Occipital Dermoid Tumor and Sinus. Annals of Plastic Surgery, 21, 465-467. http://dx.doi.org/10.1097/00000637-198811000-00012

[15] Wallace, D., Tress, B. and Kwan, P.F. (2008) Radiologically Atypical Congenital Posterior Fossa Dermoid Cyst Presenting Late in Life. Journal of Clinical Neuroscience, 15, 835-838. http://dx.doi.org/10.1016/j.jocn.2007.03.023 